\title{
Numerical Solutions of Stochastic Differential Delay Equations with Poisson Random Measure under the Generalized Khasminskii-Type Conditions
}

\author{
Minghui Song and Hui Yu \\ Department of Mathematics, Harbin Institute of Technology, Harbin 150001, China \\ Correspondence should be addressed to Minghui Song, songmh@lsec.cc.ac.cn
}

Received 3 April 2012; Accepted 22 May 2012

Academic Editor: Zhenya Yan

Copyright (C) 2012 M. Song and H. Yu. This is an open access article distributed under the Creative Commons Attribution License, which permits unrestricted use, distribution, and reproduction in any medium, provided the original work is properly cited.

The Euler method is introduced for stochastic differential delay equations (SDDEs) with Poisson random measure under the generalized Khasminskii-type conditions which cover more classes of such equations than before. The main aims of this paper are to prove the existence of global solutions to such equations and then to investigate the convergence of the Euler method in probability under the generalized Khasminskii-type conditions. Numerical example is given to indicate our results.

\section{Introduction}

To take into consideration stochastic effects such as corporate defaults, operational failures, market crashes or central bank announcements in financial market, the research on stochastic differential equations (SDEs) with Poisson random measure (see [1,2]) is important, since Merton initiated the model of such equations in 1976 (see [3]). Due to the rate of change of financial dynamics system depending on its past history, SDDE with Poisson random measure (see $[4,5])$, the case we propose and consider in this work, is meaningful.

Since there is no explicit solution for an SDDE with Poisson random measure, one needs, in general, numerical methods which can be classified into strong approximations and weak approximations (see [6-8]).

We here give an overview of the results on the strong approximations of differential equation driven by Wiener process and Poisson random measure. Platen [9] presented a convergence theorem with order $\gamma \in\{0.5,1,1.5, \ldots\}$ and originally introduced the jumpadapted methods which are based on all the jump times. Moreover, Bruti-Liberati and Platen 
(see [10]) get the jump-adapted order 1.5 scheme, and they also construct the derivative free or implicit jump-adapted schemes with desired order of strong convergence. In [11], for a class of pure jump systems, the order of Taylor schemes is given under weaker conditions than the current literature. In $[7,10]$, Bruti-Liberati and Platen present the drift-implicit schemes which have order $\gamma \in\{0.5,1\}$. Recently, [8] develops adaptive time stepping algorithms based on a jump augmented Monte Carlo Euler-Maruyama method, which achieve a prescribed precision. Mao [4] presents the convergence of numerical solutions for variable delay differential equations with Poisson random measure. In [12], the improved Runge-Kutta methods are presented to improve the accuracy behaviour of problems with small noise for SDEs driven by Poisson random measure. Clearly, the results above require that the SDEs with poisson random measure satisfy the global Lipschitz conditions and the linear growth conditions. In [5], the Euler scheme is proved to converge to the analytic solution for SDDEs with Wiener process and Poisson random measure under weaker conditions than the global Lipschitz condition and the linear growth condition.

However, there are many SDDEs with Poisson random measure, especially highly nonlinear equations, which do not satisfy the above-mentioned conditions and classical Khasminskii-type conditions (see [13-15]). And in Section 5, we give such highly nonlinear equation. Our work is motivated by [16] in which the generalized Khasminskii-type conditions are applied to SDDEs with Wiener process. The main contribution in our paper is to present Euler method for SDDEs with Poisson random measure under the generalized Khasminskii-type conditions which cover more classes of these equations than all the mentioned classical conditions above.

Our work is organized as follows. In Section 2, the properties of SDDEs with Poisson random measure are given under the generalized Khasminskii-type conditions. In Section 3, Euler method is analyzed under such conditions. In Section 4, we present the convergence in probability of the Euler method. In Section 5, an example is given.

\section{The Generalized Khasminskii-Type Conditions for SDDEs with Poisson Random Measure}

\subsection{Problem's Setting}

Throughout this paper, unless otherwise specified, we use the following notations. Let $|\cdot|$ be the Euclidean norm in $\mathbf{R}^{d}, d \in \mathbf{N}$. Let $u_{1} \vee u_{2}=\max \left\{u_{1}, u_{2}\right\}$ and $u_{1} \wedge u_{2}=\min \left\{u_{1}, u_{2}\right\}$. If $A$ is a vector or matrix, its transpose is denoted by $A^{T}$. If $A$ is a matrix, its trace norm is denoted by $|A|=\sqrt{\operatorname{trace}\left(A^{T} A\right)}$. Let $\tau>0$ and $\mathbf{R}_{+}=[0, \infty)$. Let $C\left([-\tau, 0] ; \mathbf{R}^{d}\right)$ denote the family of continuous functions from $[-\tau, 0]$ to $\mathbf{R}^{d}$ with the norm $|\varphi|=\sup _{-\tau \leq \theta \leq 0}|\varphi(\theta)|$. Denote by $C\left(\mathbf{R}^{d} ; \mathbf{R}_{+}\right)$the family of continuous functions from $\mathbf{R}^{d}$ to $\mathbf{R}_{+}$. Let $C^{2}\left(\mathbf{R}^{d} ; \mathbf{R}_{+}\right)$denote the family of continuously two times differentiable $\mathbf{R}_{+}$-valued functions from $\mathbf{R}^{d}$ to $\mathbf{R}_{+}$. [z] denotes the largest integer which is less than or equal to $z$ in $\mathbf{R}$. $I_{\mathcal{A}}$ denotes the indicator function of a set $A$.

The following $d$-dimensional SDDE with Poisson random measure is considered in our paper:

$$
\begin{aligned}
d x(t)= & a\left(x\left(t^{-}\right), x\left((t-\tau)^{-}\right)\right) d t+b\left(x\left(t^{-}\right), x\left((t-\tau)^{-}\right)\right) d W(t) \\
& +\int_{\varepsilon} c\left(x\left(t^{-}\right), x\left((t-\tau)^{-}\right), v\right) \tilde{p}_{\phi}(d v \times d t)
\end{aligned}
$$


for $t>0$, where $\tilde{p}_{\phi}(d v \times d t):=p_{\phi}(d v \times d t)-\phi(d v) d t$. Here $x\left(t^{-}\right)$denotes $\lim _{s \uparrow t} x(s)$. The initial data of (2.1) is given by

$$
\{x(t):-\tau \leq t \leq 0\}=\xi(t) \in C\left([-\tau, 0] ; \mathbf{R}^{d}\right),
$$

where $x\left(-\tau^{-}\right)=x(-\tau)$.

The drift coefficient $a: \mathbf{R}^{d} \times \mathbf{R}^{d} \rightarrow \mathbf{R}^{d}$, the diffusion coefficient $b: \mathbf{R}^{d} \times \mathbf{R}^{d} \rightarrow \mathbf{R}^{d \times m_{0}}$, and the jump coefficient $c: \mathbf{R}^{d} \times \mathbf{R}^{d} \times \varepsilon \rightarrow \mathbf{R}^{d}$ are assumed to be Borel measurable functions and the coefficients are sufficiently smooth.

The randomness in (2.1) is generated by the following (see [8]). An $m_{0}$-dimensional Wiener process $W=\left\{W(t)=\left(W^{1}(t), \ldots, W^{m_{0}}(t)\right)^{T}\right\}$ with independent scalar components is defined on a filtered probability space $\left(\Omega^{W}, \boldsymbol{F}^{W},\left(\boldsymbol{F}_{t}^{W}\right)_{t>0}, \mathbf{P}^{W}\right)$. A Poisson random measure $p_{\phi}(\omega, d v \times d t)$ is on $\Omega^{J} \times \varepsilon \times[0, \infty)$, where $\varepsilon \subseteq \mathbf{R}^{r} \backslash\{0\}$ with $r \in \mathbf{N}$, and its deterministic compensated measure $\phi(d v) d t=\lambda f(v) d v d t . f(v)$ is a probability density, and we require finite intensity $\lambda=\phi(\varepsilon)<\infty$. The Poisson random measure is defined on a filtered probability space $\left(\Omega^{J}, \mathscr{F}^{J},\left(\mathscr{F}_{t}^{J}\right)_{t \geq 0}, \mathbf{P}^{J}\right)$. The process $x(t)$ is thus defined on a product space $\left(\Omega, \mathcal{F},\left(\mathcal{F}_{t}\right)_{t \geq 0}, \mathbf{P}\right)$, where $\Omega=\Omega^{W} \times \Omega^{J}, \mathcal{F}=\mathcal{F}^{W} \times \mathcal{F}^{J},\left(\mathcal{F}_{t}\right)_{t \geq 0}=\left(\mathcal{F}_{t}^{W}\right)_{t \geq 0} \times\left(\mathcal{F}_{t}^{J}\right)_{t \geq 0}, \mathbf{P}=\mathbf{P}^{W} \times \mathbf{P}^{J}$, and $\mathcal{F}_{0}$ contains all P-null sets. The Wiener process and the Poisson random measure are mutually independent.

To state the generalized Khasminskii-type conditions, we define the operator $L V$ : $\mathbf{R}^{d} \times \mathbf{R}^{d} \rightarrow \mathbf{R}$ by

$$
\begin{aligned}
L V(x, y)= & V_{x}(x) a(x, y)+\frac{1}{2} \operatorname{trace}\left(b^{\mathrm{T}}(x, y) V_{x x}(x) b(x, y)\right) \\
& +\int_{\varepsilon}\left(V(x+c(x, y, v))-V(x)-V_{x}(x) c(x, y, v)\right) \phi(d v),
\end{aligned}
$$

where

$$
V \in C^{2}\left(\mathbf{R}^{d} ; \mathbf{R}_{+}\right), \quad V_{x}=\left(\frac{\partial V(x)}{\partial x_{1}}, \ldots, \frac{\partial V(x)}{\partial x_{d}}\right), \quad V_{x x}=\left(\frac{\partial^{2} V(x)}{\partial x_{i} \partial x_{j}}\right)_{d \times d}
$$

Now the generalized Khasminskii-type conditions are given by the following assumptions.

Assumption 2.1. For each integer $k \geq 1$, there exists a positive constant $C_{k}$, dependent on $k$, such that

$$
|a(x, y)-a(\bar{x}, \bar{y})|^{2} \vee|b(x, y)-b(\bar{x}, \bar{y})|^{2} \leq C_{k}\left(|x-y|^{2}+|\bar{x}-\bar{y}|^{2}\right)
$$

for $x, y \in \mathbf{R}^{d}$ with $|x| \vee|y| \leq k$. And there exists a positive constant $C$ such that

$$
\int_{\varepsilon}|c(x, y, v)-c(\bar{x}, \bar{y}, v)|^{2} \phi(d v) \leq C\left(|x-y|^{2}+|\bar{x}-\bar{y}|^{2}\right),
$$

for $x, y \in \mathbf{R}^{d}$. 
Assumption 2.2. There are two functions $V \in C^{2}\left(\mathbf{R}^{d} ; \mathbf{R}_{+}\right)$and $U \in C\left(\mathbf{R}^{d} ; \mathbf{R}_{+}\right)$as well as two positive constants $\mu_{1}$ and $\mu_{2}$ such that

$$
\begin{gathered}
\lim _{|x| \rightarrow \infty} V(x)=\infty \\
L V(x, y) \leq \mu_{1}(1+V(x)+V(y)+U(y))-\mu_{2} U(x),
\end{gathered}
$$

for all $(x, y) \in \mathbf{R}^{d} \times \mathbf{R}^{d}$.

Assumption 2.3. There exists a positive constant $\bar{C}$ such that

$$
\int_{\varepsilon}|c(0,0, v)|^{2} \phi(d v) \leq \bar{C}
$$

Assumption 2.4. There exists a positive constant $L$ such that the initial data (2.2) satisfies

$$
|\xi(t)-\xi(s)| \leq L|t-s|^{1 / 2}, \quad \text { for }-\tau \leq t, s \leq 0 .
$$

\subsection{The Existence of Global Solutions}

In this section, we analyze the existence and the property of the global solution to (2.1) under Assumptions 2.1, 2.2, and 2.4.

In order to demonstrate the existence of the global solution to (2.1), we redefine the following concepts mainly according to $[17,18]$.

Definition 2.5. Let $\{x(t)\}_{t \geq-\tau}$ be an $\mathbf{R}^{d}$-valued stochastic process. The process is said to be càdlàg if it is right continuous and for almost all $\omega \in \Omega$ the left $\operatorname{limit}_{\lim } \operatorname{lit}_{s} x(s)$ exists and is finite for all $t>-\tau$.

Definition 2.6. Let $\sigma_{\infty}$ be a stopping time such that $0 \leq \sigma_{\infty} \leq T$ a.s. An $\mathbf{R}^{d}$-valued, $\boldsymbol{F}_{t}$-adapted, and càdlàg process $\left\{x(t):-\tau \leq t<\sigma_{\infty}\right\}$ is called a local solution of $(2.1)$ if $x(t)=\xi(t)$ on $t \in[-\tau, 0]$ and, moreover, there is a nondecreasing sequence $\left\{\sigma_{k}\right\}_{k \geq 1}$ of stopping times such that $0 \leq \sigma_{k} \uparrow \sigma_{\infty}$ a.s. and

$$
\begin{aligned}
x\left(t \wedge \sigma_{k}\right)= & x(0)+\int_{0}^{t \wedge \sigma_{k}} a\left(x\left(s^{-}\right), x\left((s-\tau)^{-}\right)\right) d s+\int_{0}^{t \wedge \sigma_{k}} b\left(x\left(s^{-}\right), x\left((s-\tau)^{-}\right)\right) d W(s) \\
& +\int_{0}^{t \wedge \sigma_{k}} \int_{\varepsilon} c\left(x\left(s^{-}\right), x\left((s-\tau)^{-}\right), v\right) \tilde{p}_{\phi}(d v \times d s)
\end{aligned}
$$

holds for any $t \in[0, T]$ and $k \geq 1$ with probability 1 . If, furthermore,

$$
\lim _{t \rightarrow \sigma_{\infty}} \sup |x(t)|=\infty \quad \text { whenever } \sigma_{\infty}<T
$$

then it is called a maximal local solution of (2.1) and $\sigma_{\infty}$ is called the explosion time. A local solution $\left\{x(t):-\tau \leq t<\sigma_{\infty}\right\}$ to (2.1) is called a global solution if $\sigma_{\infty}=\infty$. 
Lemma 2.7. Under Assumptions 2.1 and 2.4, for any given initial data (2.2), there is a unique maximal local solution to (2.1).

Proof. From Assumption 2.4, for the initial data (2.2), we have

$$
\max _{-\tau \leq t \leq 0}|\xi(t)| \leq \max _{-\tau \leq t \leq 0}|\xi(t)-\xi(0)|+|\xi(0)| \leq L \sqrt{\tau}+|\xi(0)| .
$$

For each integer $k \geq[L \sqrt{\tau}+|\xi(0)|]+1$, we define

$$
z^{[k]}=\frac{|z| \wedge k}{|z|} z, \quad 0^{[k]}=0,
$$

for $z \in \mathbf{R}^{d}$. And then we define the truncation functions

$$
a_{k}(x, y)=a\left(x^{[k]}, y^{[k]}\right), \quad b_{k}(x, y)=b\left(x^{[k]}, y^{[k]}\right), \quad c_{k}(x, y, v)=c(x, y, v),
$$

for $x, y \in \mathbf{R}^{d}$ and each $k \geq[L \sqrt{\tau}+|\xi(0)|]+1$. Moreover, we define the following equation:

$$
\begin{aligned}
d x_{k}(t)= & a_{k}\left(x_{k}\left(t^{-}\right), x_{k}\left((t-\tau)^{-}\right)\right) d t+b_{k}\left(x_{k}\left(t^{-}\right), x_{k}\left((t-\tau)^{-}\right)\right) d W(t) \\
& +\int_{\varepsilon} c_{k}\left(x_{k}\left(t^{-}\right), x_{k}\left((t-\tau)^{-}\right), v\right) \tilde{p}_{\phi}(d v \times d t),
\end{aligned}
$$

on $t \in[0, T]$ with initial data $x_{k}(t)=\xi(t)$ on $t \in[-\tau, 0]$. Obviously, the equation satisfies the global Lipschitz conditions and the linear growth conditions. Therefore according to [4], there is a unique global solution $x_{k}(t)$ to (2.16) and its solution is a càdlàg process (see [17]). We define the stopping time

$$
\sigma_{k}=T \wedge \inf \left\{t \in[0, T]:\left|x_{k}(t)\right| \geq k\right\},
$$

for $k \geq[L \sqrt{\tau}+|\xi(0)|]+1$, and

$$
\sigma_{1}=\cdots=\sigma_{[L \sqrt{\tau}+|\xi(0)|]}=\sigma_{[L \sqrt{\tau}+|\xi(0)|]+1}
$$

where we set $\inf \phi=\infty$ (as usual $\phi$ denotes the empty set) throughout our paper. We can easily get

$$
x_{k}(t)=x_{k+1}(t), \quad-\tau \leq t \leq \sigma_{k},
$$

which means $\left\{\sigma_{k}\right\}_{k \geq 1}$ is a nondecreasing sequence and then let $\lim _{k \rightarrow \infty} \sigma_{k}=\sigma_{\infty}$ a.s. Now, we define $\left\{x(t):-\tau \leq t<\sigma_{\infty}\right\}$ with $x(t)=\xi(t)$ on $t \in[-\tau, 0]$ and

$$
x(t)=x_{k}(t), \quad t \in\left[\sigma_{k-1}, \sigma_{k}\right), k \geq 1,
$$


where $\sigma_{0}=0$. And from (2.16) and (2.19), we can also obtain

$$
\begin{aligned}
x\left(t \wedge \sigma_{k}\right)= & x_{k}\left(t \wedge \sigma_{k}\right) \\
= & x_{0}+\int_{0}^{t \wedge \sigma_{k}} a\left(x\left(s^{-}\right), x\left((s-\tau)^{-}\right)\right) d s+\int_{0}^{t \wedge \sigma_{k}} b\left(x\left(s^{-}\right), x\left((s-\tau)^{-}\right)\right) d W(s) \\
& +\int_{0}^{t \wedge \sigma_{k}} \int_{\varepsilon} c\left(x\left(s^{-}\right), x\left((s-\tau)^{-}\right), v\right) \tilde{p}_{\phi}(d v \times d s),
\end{aligned}
$$

for any $t \in[0, T]$ and $k \geq 1$ with probability 1 . Moreover, if $\sigma_{\infty}<T$, then

$$
\lim _{t \rightarrow \sigma_{\infty}} \sup |x(t)| \geq \lim _{k \rightarrow \infty} \sup \left|x\left(\sigma_{k}\right)\right|=\lim _{k \rightarrow \infty} \sup \left|x_{k}\left(\sigma_{k}\right)\right|=\infty .
$$

Hence $\left\{x(t):-\tau \leq t<\sigma_{\infty}\right\}$ is a maximal local solution to (2.1).

To show the uniqueness of the solution to (2.1), let $\left\{\bar{x}(t):-\tau \leq t<\bar{\sigma}_{\infty}\right\}$ be another maximal local solution. As the same proof as Theorem 2.8 in [17], we infer that

$$
\mathbf{P}\left(x(t, \omega)=\bar{x}(t, \omega),(t, \omega) \in\left[-\tau, \sigma_{k} \wedge \bar{\sigma}_{k}\right) \times \Omega\right)=1, \quad k \geq 1 .
$$

Hence by $k \rightarrow \infty$, we get

$$
\mathbf{P}\left(x(t, \omega)=\bar{x}(t, \omega),(t, \omega) \in\left[-\tau, \sigma_{\infty} \wedge \bar{\sigma}_{\infty}\right) \times \Omega\right)=1 .
$$

Therefore $x(t)$ is a unique local solution and then it is a unique maximal local solution to (2.1).

So we complete the whole proof.

Now, the existence of the global solution to (2.1) is shown in the following theorem.

Theorem 2.8. Under Assumptions 2.1, 2.2, and 2.4, for any given initial data (2.2), there is a unique global solution $x(t)$ to $(2.1)$ on $t \in[-\tau, \infty)$.

Proof. According to Lemma 2.7, there exists a unique maximal local solution to (2.1) on $\left[-\tau, \sigma_{\infty}\right)$. Hence in order to show that this local solution is a global one, we only need to demonstrate $\sigma_{\infty}=\infty$ a.s. Using Itô's formula (see [1]) to $V(x(t))$, we have

$$
\begin{aligned}
d V(x(t))=( & V_{x}\left(x\left(t^{-}\right)\right) a\left(x\left(t^{-}\right), x\left((t-\tau)^{-}\right)\right) \\
& \left.+\frac{1}{2} \operatorname{trace}\left(b^{\mathrm{T}}\left(x\left(t^{-}\right), x\left((t-\tau)^{-}\right)\right) V_{x x}\left(x\left(t^{-}\right)\right) b\left(x\left(t^{-}\right), x\left((t-\tau)^{-}\right)\right)\right)\right) d t \\
& +\int_{\varepsilon}\left(V\left(x\left(t^{-}\right)+c\left(x\left(t^{-}\right), x\left((t-\tau)^{-}\right), v\right)\right)\right. \\
& \left.-V\left(x\left(t^{-}\right)\right)-V_{x}\left(x\left(t^{-}\right)\right) c\left(x\left(t^{-}\right), x\left((t-\tau)^{-}\right), v\right)\right) \phi(d v) d t \\
+ & V_{x}\left(x\left(t^{-}\right)\right) b\left(x\left(t^{-}\right), x\left((t-\tau)^{-}\right)\right) d W(t) \\
+ & \int_{\varepsilon}\left(V\left(x\left(t^{-}\right)+c\left(x\left(t^{-}\right), x\left((t-\tau)^{-}\right), v\right)\right)-V\left(x\left(t^{-}\right)\right)\right) \tilde{p}_{\phi}(d v \times d t)
\end{aligned}
$$


Abstract and Applied Analysis

$$
\begin{aligned}
= & L V\left(x\left(t^{-}\right), x\left((t-\tau)^{-}\right)\right) d t+V_{x}\left(x\left(t^{-}\right)\right) b\left(x\left(t^{-}\right), x\left((t-\tau)^{-}\right)\right) d W(t) \\
& +\int_{\varepsilon}\left(V\left(x\left(t^{-}\right)+c\left(x\left(t^{-}\right), x\left((t-\tau)^{-}\right), v\right)\right)-V\left(x\left(t^{-}\right)\right)\right) \tilde{p}_{\phi}(d v \times d t),
\end{aligned}
$$

for $t \in\left[0, \sigma_{\infty}\right)$.

Our proof is divided into the following steps.

Step 1. For any integer $k \geq[L \sqrt{\tau}+|\xi(0)|]+1$ and $0 \leq t \leq \tau$, by taking integration and expectations and using Assumption 2.2 to (2.25), we get

$$
\begin{aligned}
& \mathrm{E} V\left(x\left(t \wedge \sigma_{k}\right)\right)-\mathbf{E} V(x(0)) \\
& \quad \leq \mathrm{E} \int_{0}^{t \wedge \sigma_{k}}\left(\mu_{1}\left(1+V\left(x\left(s^{-}\right)\right)+V\left(x\left((s-\tau)^{-}\right)\right)+U\left(x\left((s-\tau)^{-}\right)\right)\right)-\mu_{2} U\left(x\left(s^{-}\right)\right)\right) d s
\end{aligned}
$$

which means

$$
\mathbf{E} V\left(x\left(t \wedge \sigma_{k}\right)\right) \leq C_{1}+\mu_{1} \mathbf{E} \int_{0}^{t \wedge \sigma_{k}} V\left(x\left(s^{-}\right)\right) d s-\mu_{2} \mathbf{E} \int_{0}^{t \wedge \sigma_{k}} U\left(x\left(s^{-}\right)\right) d s
$$

where

$$
C_{1}=V(x(0))+\mu_{1} \tau+\mu_{1} \int_{-\tau}^{0} V(\xi(s)) d s+\mu_{1} \int_{-\tau}^{0} U(\xi(s)) d s<\infty
$$

From (2.27), we obtain

$$
\begin{aligned}
\mathbf{E} V\left(x\left(t \wedge \sigma_{k}\right)\right) & \leq C_{1}+\mu_{1} \mathbf{E} \int_{0}^{t \wedge \sigma_{k}} V\left(x\left(s^{-}\right)\right) d s \\
& \leq C_{1}+\mu_{1} \int_{0}^{t} \mathbf{E} V\left(x\left(s \wedge \sigma_{k}^{-}\right)\right) d s,
\end{aligned}
$$

by the Gronwall inequality (see [18]), which leads to

$$
\mathrm{E} V\left(x\left(t \wedge \sigma_{k}\right)\right) \leq C_{1} e^{\mu_{1} \tau},
$$

for $0 \leq t \leq \tau$ and $k \geq[L \sqrt{\tau}+|\xi(0)|]+1$. Let

$$
\varsigma_{k}=\inf _{|x| \geq k, 0 \leq t<\infty} V(x), \quad \text { for } k \geq[L \sqrt{\tau}+|\xi(0)|]+1 .
$$

Therefore, from (2.30), we have

$$
\varsigma_{k} \mathbf{P}\left(\sigma_{k} \leq \tau\right) \leq \mathbf{E}\left(V\left(x\left(\sigma_{k}\right)\right) I_{\left\{\sigma_{k} \leq \tau\right\}}\right) \leq \mathbf{E} V\left(x\left(\tau \wedge \sigma_{k}\right)\right) \leq C_{1} e^{\mu_{1} \tau},
$$


by taking $k \rightarrow \infty$, which gives

$$
\mathbf{P}\left(\sigma_{\infty} \leq \tau\right)=0 .
$$

Hence we get

$$
\mathbf{P}\left(\sigma_{\infty}>\tau\right)=1
$$

It thus follows from (2.30) and (2.34) that

$$
\mathrm{E} V(x(t)) \leq C_{1} e^{\mu_{1} \tau}, \quad 0 \leq t \leq \tau
$$

by taking $k \rightarrow \infty$.

Moreover, from (2.27), we get

$$
\begin{aligned}
\mathrm{E} \int_{0}^{\tau \wedge \sigma_{k}} U\left(x\left(s^{-}\right)\right) d s & \leq \mu_{2}^{-1}\left(C_{1}+\mu_{1} \mathrm{E} \int_{0}^{\tau \wedge \sigma_{k}} V\left(x\left(s^{-}\right)\right) d s\right) \\
& \leq \mu_{2}^{-1}\left(C_{1}+\mu_{1} \int_{0}^{\tau} \mathrm{E} V\left(x\left(s \wedge \sigma_{k}^{-}\right)\right) d s\right),
\end{aligned}
$$

by taking $k \rightarrow \infty$, which gives

$$
\mathrm{E} \int_{0}^{\tau} U(x(s)) d s \leq \mu_{2}^{-1}\left(C_{1}+\tau \mu_{1} C_{1} e^{\mu_{1} \tau}\right)<\infty,
$$

where (2.34) and (2.35) are used.

Step 2. For any integer $k \geq[L \sqrt{\tau}+|\xi(0)|]+1$ and $0 \leq t \leq 2 \tau$, the similar analysis as above gives

$$
\mathbf{E} V\left(x\left(t \wedge \sigma_{k}\right)\right) \leq C_{2}+\mu_{1} \mathbf{E} \int_{0}^{t \wedge \sigma_{k}} V\left(x\left(s^{-}\right)\right) d s-\mu_{2} \mathbf{E} \int_{0}^{t \wedge \sigma_{k}} U\left(x\left(s^{-}\right)\right) d s,
$$

where

$$
\begin{aligned}
C_{2}= & V(x(0))+2 \mu_{1} \tau+\mu_{1} \int_{-\tau}^{0} V(\xi(s)) d s+\mu_{1} \int_{-\tau}^{0} U(\xi(s)) d s \\
& +\mu_{1} \mathbf{E} \int_{0}^{\tau} V(x(s)) d s+\mu_{1} \mathbf{E} \int_{0}^{\tau} U(x(s)) d s<\infty,
\end{aligned}
$$

from (2.35) and (2.37).

Thus,

$$
\mathbf{E} V\left(x\left(t \wedge \sigma_{k}\right)\right) \leq C_{2}+\mu_{1} \mathbf{E} \int_{0}^{t} V\left(x\left(s \wedge \sigma_{k}^{-}\right)\right) d s
$$


which gives

$$
\mathrm{EV}\left(x\left(t \wedge \sigma_{k}\right)\right) \leq C_{2} e^{2 \mu_{1} \tau},
$$

for $0 \leq t \leq 2 \tau$ and $k \geq[L \sqrt{\tau}+|\xi(0)|]+1$. Hence we get

$$
\varsigma_{k} \mathbf{P}\left(\sigma_{k} \leq 2 \tau\right) \leq \mathbf{E} V\left(x\left(2 \tau \wedge \sigma_{k}\right)\right) \leq C_{2} e^{2 \mu_{1} \tau},
$$

by taking $k \rightarrow \infty$, which implies

$$
\mathbf{P}\left(\sigma_{\infty} \leq 2 \tau\right)=0,
$$

that is,

$$
\mathbf{P}\left(\sigma_{\infty}>2 \tau\right)=1 .
$$

Moreover, by taking $k \rightarrow \infty$ to (2.41), we then get

$$
\mathbf{E} V(x(t)) \leq C_{2} e^{2 \mu_{1} \tau}, \quad 0 \leq t \leq 2 \tau .
$$

Therefore, from (2.38), (2.44), and (2.45), we have

$$
\mathrm{E} \int_{0}^{2 \tau} U(x(s)) d s \leq \mu_{2}^{-1}\left(C_{2}+2 \tau \mu_{1} C_{1} e^{2 \mu_{1} \tau}\right)<\infty .
$$

Step 3. So for any $i \in \mathbf{N}$, we repeat the similar analysis as above and then obtain

$$
\begin{gathered}
\mathbf{P}\left(\sigma_{\infty}>i \tau\right)=1 \\
\mathbf{E} V(x(t)) \leq C_{i} e^{i \mu_{1} \tau}, \quad 0 \leq t \leq i \tau \\
\mathbf{E} \int_{0}^{\tau} U(x(s)) d s \leq \mu_{2}^{-1}\left(C_{i}+i \tau \mu_{1} C_{i} e^{i \mu_{1} \tau}\right)<\infty
\end{gathered}
$$

where

$$
C_{i}=V(x(0))+\mu_{1} \mathbf{E} \int_{-\tau}^{(i-1) \tau}(1+V(x(s))+U(x(s))) d s<\infty .
$$

So we can get $\mathbf{P}\left(\sigma_{\infty}=\infty\right)=1$ and the required result follows.

In the following lemma, we show that the solution of (2.1) remains in a compact set with a large probability. 
Lemma 2.9. Under Assumptions 2.1, 2.2, and 2.4, for any pair of $\epsilon \in(0,1)$ and $T>0$, there exists a sufficiently large integer $k^{*}$, dependent on $\epsilon$ and $T$, such that

$$
\mathbf{P}\left(\sigma_{k} \leq T\right) \leq \epsilon, \quad \forall k \geq k^{*},
$$

where $\sigma_{k}$ is defined in Lemma 2.7.

Proof. According to Theorem 2.8, we can get

$$
\mathbf{E V}\left(x\left(T \wedge \sigma_{k}\right)\right) \leq C_{i} e^{i \mu_{1} \tau},
$$

for $i$ large enough to $i \tau \geq T$ and $k \geq[L \sqrt{\tau}+|\xi(0)|]+1$. Therefore, we have

$$
\varsigma_{k} \mathbf{P}\left(\sigma_{k} \leq T\right) \leq \mathbf{E}\left(V\left(x\left(\sigma_{k}\right)\right) I_{\left\{\sigma_{k} \leq T\right\}}\right) \leq \mathbf{E} V\left(x\left(T \wedge \sigma_{k}\right)\right) \leq C_{i} e^{i \mu_{1} \tau},
$$

where

$$
S_{k}=\inf _{|x| \geq k} V(x) \quad \text { for } k \geq[L \sqrt{\tau}+|\xi(0)|]+1 .
$$

Under (2.7) in Assumption 2.2, there exists a sufficiently large integer $k^{*}$ such that

$$
\mathbf{P}\left(\sigma_{k} \leq T\right) \leq \frac{C_{i} e^{i \mu_{1} \tau}}{\varsigma_{k}} \leq \epsilon, \quad \forall k \geq k^{*}
$$

So we complete the proof.

\section{The Euler Method}

In this section, we introduce the Euler method to (2.1) under Assumptions 2.1, 2.2, 2.3, and 2.4 .

Given a step size $\Delta t=\tau / m \in(0,1), m \in \mathbf{N}$, the Euler method applied to (2.1) computes approximation $X_{n} \approx x\left(t_{n}\right)$, where $t_{n}=n \Delta t$ for $n=-m,-(m-1), \ldots,-1,0,1, \ldots$, by setting

$$
X_{n}=\xi(n \Delta t) \quad \text { for } n=-m,-(m-1), \ldots,-1,0,
$$

and forming

$$
\begin{aligned}
X_{n+1}= & X_{n}+a\left(X_{n}, X_{n-m}\right) \Delta t+b\left(X_{n}, X_{n-m}\right) \Delta W_{n} \\
& +\int_{t_{n}}^{t_{n+1}} \int_{\varepsilon} c\left(X_{n}, X_{n-m}, v\right) \tilde{p}_{\phi}(d v \times d t),
\end{aligned}
$$

for $n=0,1, \ldots$, where $\Delta W_{n}=W\left(t_{n+1}\right)-W\left(t_{n}\right)$. 
The continuous-time Euler method $\bar{X}(t)$ on $t \in[-\tau, \infty)$ is then defined by

$$
\begin{gathered}
\bar{X}(t):=\xi(t) \quad \text { for } t \in[-\tau, 0], \\
\bar{X}(t):=X_{0}+\int_{0}^{t} a(Z(s), Z(s-\tau)) d s+\int_{0}^{t} b(Z(s), Z(s-\tau)) d W(s) \\
+\int_{0}^{t} \int_{\varepsilon} c(Z(s), Z(s-\tau), v) \tilde{p}_{\phi}(d v \times d s),
\end{gathered}
$$

for $t \geq 0$, where

$$
Z(t)=\sum_{n=-m}^{\infty} X_{n} I_{[n \Delta t,(n+1) \Delta t)}(t) \quad \text { for } t \in[-\tau, \infty)
$$

Actually, we can see in [11] that $p_{\phi}=\left\{p_{\phi}(t):=p_{\phi}(\varepsilon \times[0, t])\right\}$ is a process that counts the number of jumps until some given time. The Poisson random measure $p_{\phi}(d v \times d t)$ generates a sequence of pairs $\left\{\left(l_{i}, \xi_{i}\right), i \in\left\{1,2, \ldots, p_{\phi}(T)\right\}\right\}$ for a given finite positive constant $T$ if $\lambda<\infty$. Here $\left\{\iota_{i}: \Omega \rightarrow \mathbf{R}_{+}, i \in\left\{1,2, \ldots, p_{\phi}(T)\right\}\right\}$ is a sequence of increasing nonnegative random variables representing the jump times of a standard Poisson process with intensity $\lambda$, and $\left\{\xi_{i}\right.$ : $\left.\Omega \rightarrow \varepsilon, i \in\left\{1,2, \ldots, p_{\phi}(T)\right\}\right\}$ is a sequence of independent identically distributed random variables, where $\xi_{i}$ is distributed according to $\phi(d v) / \phi(\varepsilon)$. Then (3.2) can equivalently be of the following form:

$$
\begin{aligned}
X_{n+1}= & X_{n}+\left(a\left(X_{n}, X_{n-m}\right)-\int_{\varepsilon} c\left(X_{n}, X_{n-m}, v\right) \phi(d v)\right) \Delta t \\
& +b\left(X_{n}, X_{n-m}\right) \Delta W_{n}+\sum_{i=p_{\phi}\left(t_{n}\right)+1}^{p_{\phi}\left(t_{n+1}\right)} c\left(X_{n}, X_{n-m}, \xi_{i}\right) .
\end{aligned}
$$

In order to analyze the Euler method, we will give two lemmas.

The first lemma shows the close relation between the continuous-time Euler solution (3.4) and its step function $Z(t)$.

Lemma 3.1. Suppose Assumptions 2.1 and 2.3 hold. Then for any $T>0$, there exists a positive constant $K_{1}(k)$, dependent on integer $k$ and independent of $\Delta t$, such that for all $\Delta t \in(0,1)$ the continuous-time Euler method (3.4) satisfies

$$
\mathrm{E}|\bar{X}(t)-Z(t)|^{2} \leq K_{1}(k) \Delta t
$$

for $0 \leq t \leq T \wedge \sigma_{k} \wedge \rho_{k}$ and $k \geq[L \sqrt{\tau}+|\xi(0)|]+1$, where $\sigma_{k}$ is defined in Lemma 2.7 and $\rho_{k}=\inf \{t \geq$ $0:|\bar{X}(t)| \geq k\}$. 
Proof. For $0 \leq t \leq T \wedge \sigma_{k} \wedge \rho_{k}$ and $k \geq[L \sqrt{\tau}+|\xi(0)|]+1$, there is an integer $n$ such that $t \in\left[t_{n}, t_{n+1}\right)$. Thus it follows from (3.2) that

$$
\begin{aligned}
\bar{X}(t)-Z(t)= & X_{n}+\int_{t_{n}}^{t} a(Z(s), Z(s-\tau)) d s+\int_{t_{n}}^{t} b(Z(s), Z(s-\tau)) d W(s) \\
& +\int_{t_{n}}^{t} \int_{\varepsilon} c(Z(s), Z(s-\tau), v) \tilde{p}_{\phi}(d v \times d s)-X_{n} .
\end{aligned}
$$

Therefore, by taking expectations and the Cauchy-Schwarz inequality and using the martingale properties of $d W(t)$ and $\tilde{p}_{\phi}(d v \times d t)$, we get

$$
\begin{aligned}
& \mathrm{E}|\overline{\mathrm{X}}(t)-Z(t)|^{2} \\
& \leq 3 \mathrm{E}\left|\int_{t_{n}}^{t} a(Z(s), Z(s-\tau)) d s\right|^{2}+3 \mathrm{E}\left|\int_{t_{n}}^{t} b(Z(s), Z(s-\tau)) d W(s)\right|^{2} \\
& \quad+3 \mathrm{E}\left|\int_{t_{n}}^{t} \int_{\varepsilon} c(Z(s), Z(s-\tau), v) \tilde{p}_{\phi}(d v \times d s)\right|^{2} \\
& \leq 3 \Delta t \mathrm{E} \int_{t_{n}}^{t}|a(Z(s), Z(s-\tau))|^{2} d s+3 \mathrm{E} \int_{t_{n}}^{t}|b(Z(s), Z(s-\tau))|^{2} d s \\
& \quad+3 \mathrm{E} \int_{t_{n}}^{t} \int_{\varepsilon}|c(Z(s), Z(s-\tau), v)|^{2} \phi(d v) d s,
\end{aligned}
$$

where the inequality $\left|u_{1}+u_{2}+u_{3}\right|^{2} \leq 3\left|u_{1}\right|^{2}+3\left|u_{2}\right|^{2}+3\left|u_{3}\right|^{2}$ for $u_{1}, u_{2}, u_{3} \in \mathbf{R}^{d}$ is used. Therefore, by applying Assumption 2.1, we get

$$
\begin{gathered}
\mathbf{E} \int_{t_{n}}^{t}|a(Z(s), Z(s-\tau))|^{2} d s \\
\leq 2 \mathrm{E} \int_{t_{n}}^{t}|a(Z(s), Z(s-\tau))-a(0,0)|^{2} d s+2 \mathbf{E} \int_{t_{n}}^{t}|a(0,0)|^{2} d s \\
\leq 2 C_{k} \mathbf{E} \int_{t_{n}}^{t}\left(|Z(s)|^{2}+|Z(s-\tau)|^{2}\right) d s+2|a(0,0)|^{2} \Delta t \\
\leq 4 k^{2} C_{k} \Delta t+2|a(0,0)|^{2} \Delta t \\
\quad \mathbf{E} \int_{t_{n}}^{t}|b(Z(s), Z(s-\tau))|^{2} d s \leq 4 k^{2} C_{k} \Delta t+2|b(0,0)|^{2} \Delta t \\
\mathbf{E} \int_{t_{n}}^{t} \int_{\varepsilon}|c(Z(s), Z(s-\tau), v)|^{2} \phi(d v) d s \leq 4 k^{2} C \Delta t+2 \Delta t \int_{\varepsilon}|c(0,0, v)|^{2} \phi(d v) .
\end{gathered}
$$


Hence by substituting (3.10) into (3.9), we get

$$
\mathbf{E}|\bar{X}(t)-Z(t)|^{2} \leq \Delta t\left(24 k^{2} C_{k}+12 k^{2} C+6|a(0,0)|^{2}+6|b(0,0)|^{2}+6 \int_{\varepsilon}|c(0,0, v)|^{2} \phi(d v)\right),
$$

for $0 \leq t \leq T \wedge \sigma_{k} \wedge \rho_{k}$ and $k \geq[L \sqrt{\tau}+|\xi(0)|]+1$.

So from Assumption 2.3, we can get the result (3.7) by choosing

$$
K_{1}(k)=24 k^{2} C_{k}+12 k^{2} C+6|a(0,0)|^{2}+6|b(0,0)|^{2}+6 \int_{\varepsilon}|c(0,0, v)|^{2} \phi(d v) .
$$

In the following lemma, we demonstrate that the solution of continuous-time Euler method (3.4) remains in a compact set with a large probability.

Lemma 3.2. Under Assumptions 2.1, 2.2, 2.3, and 2.4, for any pair of $\epsilon \in(0,1)$ and $T>0$, there exist a sufficiently large integer $k^{*}$ and a sufficiently small $\Delta t_{1}^{*}$ such that

$$
\mathbf{P}\left(\rho_{k^{*}} \leq T\right) \leq \epsilon, \quad \forall \Delta t \leq \Delta t_{1}^{*}
$$

where $\rho_{k^{*}}$ is defined in Lemma 3.1 .

Proof. Our proof is completed by the following steps.

Step 1. Using Itô's formula (see [1]) to $V(\bar{X}(t))$, for $t \geq 0$, we have

$$
\begin{aligned}
d V(\bar{X}(t))= & V_{x}(\bar{X}(t)) a(Z(t), Z(t-\tau)) \\
& \left.+\frac{1}{2} \operatorname{trace}\left(b^{\mathrm{T}}(Z(t), Z(t-\tau)) V_{x x}(\bar{X}(t)) b(Z(t), Z(t-\tau))\right)\right) d t \\
+ & \int_{\varepsilon}(V(\bar{X}(t)+c(Z(t), Z(t-\tau), v)) \\
& \left.-V(\bar{X}(t))-V_{x}(\bar{X}(t)) c(Z(t), Z(t-\tau), v)\right) \phi(d v) d t \\
+ & V_{x}(\bar{X}(t)) b(Z(t), Z(t-\tau)) d W(t) \\
+ & \int_{\varepsilon}(V(\bar{X}(t)+c(Z(t), Z(t-\tau), v))-V(\bar{X}(t))) \tilde{p}_{\phi}(d v \times d t) \\
= & L V(\bar{X}(t), \bar{X}(t-\tau)) d t+f(\bar{X}(t), \bar{X}(t-\tau), Z(t), Z(t-\tau)) d t \\
& +V_{x}(\bar{X}(t)) b(Z(t), Z(t-\tau)) d W(t) \\
& +\int_{\varepsilon}(V(\bar{X}(t)+c(Z(t), Z(t-\tau), v))-V(\bar{X}(t))) \tilde{p}_{\phi}(d v \times d t)
\end{aligned}
$$


where $f\left(x, y, Z_{1}, Z_{2}\right): \mathbf{R}^{d} \times \mathbf{R}^{d} \times \mathbf{R}^{d} \times \mathbf{R}^{d} \rightarrow \mathbf{R}$ is defined by

$$
\begin{aligned}
f\left(x, y, Z_{1}, Z_{2}\right) \\
=V_{x}(x)\left(a\left(Z_{1}, Z_{2}\right)-a(x, y)\right)+\frac{1}{2} \operatorname{trace}\left(b^{\mathrm{T}}\left(Z_{1}, Z_{2}\right) V_{x x}(x) b\left(Z_{1}, Z_{2}\right)\right) \\
\quad-\frac{1}{2} \operatorname{trace}\left(b^{\mathrm{T}}(x, y) V_{x x}(x) b(x, y)\right) \\
\quad+\int_{\varepsilon}\left(V_{x}(x) c(x, y, v)-V_{x}(x) c\left(Z_{1}, Z_{2}, v\right)\right) \phi(d v) \\
\quad+\int_{\varepsilon}\left(V\left(x+c\left(Z_{1}, Z_{2}, v\right)\right)-V(x+c(x, y, v))\right) \phi(d v) .
\end{aligned}
$$

Moreover, for $\left(x, y, Z_{1}, Z_{2}\right) \in \mathbf{R}^{d} \times \mathbf{R}^{d} \times \mathbf{R}^{d} \times \mathbf{R}^{d}$ with $|x| \vee|y| \vee\left|Z_{1}\right| \vee\left|Z_{2}\right| \leq k$, we have

$$
\begin{aligned}
f\left(x, y, Z_{1}, Z_{2}\right)= & V_{x}(x)\left(a\left(Z_{1}, Z_{2}\right)-a(x, y)\right) \\
& +\frac{1}{2} \operatorname{trace}\left(\left(b^{\mathrm{T}}\left(Z_{1}, Z_{2}\right)-b^{\mathrm{T}}(x, y)\right) V_{x x}(x) b\left(Z_{1}, Z_{2}\right)\right) \\
& +\frac{1}{2} \operatorname{trace}\left(b^{\mathrm{T}}(x, y) V_{x x}(x)\left(b\left(Z_{1}, Z_{2}\right)-b(x, y)\right)\right) \\
& +\int_{\varepsilon} V_{x}(x)\left(c(x, y, v)-c\left(Z_{1}, Z_{2}, v\right)\right) \phi(d v) \\
& +\int_{\varepsilon}\left(V\left(x+c\left(Z_{1}, Z_{2}, v\right)\right)-V(x+c(x, y, v))\right) \phi(d v) \\
\leq & L_{k}\left(\left|x-Z_{1}\right|+\left|y-Z_{2}\right|\right),
\end{aligned}
$$

where Assumptions 2.1 and 2.2 are used and $L_{k}$ is a positive constant dependent on integer $k$, intensity $\lambda$ and independent of $\Delta t$. Therefore, from (3.16), Assumption 2.4, and (3.7) in Lemma 3.1, we obtain

$$
\begin{array}{rl}
\mathbf{E} \int_{0}^{t \wedge \rho_{k}} & f(\bar{X}(s), \bar{X}(s-\tau), Z(s), Z(s-\tau)) d s \\
\quad \leq & L_{k} \mathbf{E} \int_{0}^{t \wedge \rho_{k}}|\bar{X}(s)-Z(s)| d s+L_{k} \mathbf{E} \int_{0}^{t \wedge \rho_{k}}|\bar{X}(s-\tau)-Z(s-\tau)| d s \\
\quad \leq 2 L_{k} \mathbf{E} \int_{0}^{t \wedge \rho_{k}}|\bar{X}(s)-Z(s)| d s+L_{k} \int_{-\tau}^{0}|\xi(s)-Z(s)| d s \\
\quad \leq 2 L_{k} \int_{0}^{t}\left(\mathbf{E}\left|\bar{X}\left(s \wedge \rho_{k}\right)-Z\left(s \wedge \rho_{k}\right)\right|^{2}\right)^{1 / 2} d s+L_{k} \sum_{n=-m}^{-1} \int_{t_{n}}^{t_{n+1}}\left|\xi(s)-\xi\left(t_{n}\right)\right| d s \\
\quad \leq 2 L_{k} T \sqrt{K_{1}(k) \Delta t}+L_{k} L \tau \sqrt{\Delta t}
\end{array}
$$


for $0 \leq t \leq T$ and $k \geq[L \sqrt{\tau}+|\xi(0)|]+1$. Hence by taking expectations and integration to (3.14), applying the martingale properties of $d W(t)$ and $\tilde{p}_{\phi}(d v \times d t)$, and then using (3.17) and Assumption 2.2, we obtain

$$
\begin{aligned}
& \mathbf{E} V\left(\bar{X}\left(t \wedge \rho_{k}\right)\right) \\
& =V\left(X_{0}\right)+\mathbf{E} \int_{0}^{t \wedge \rho_{k}} L V(\bar{X}(s), \bar{X}(s-\tau)) d s \\
& \quad+\mathbf{E} \int_{0}^{t \wedge \rho_{k}} f(\bar{X}(s), \bar{X}(s-\tau), Z(s), Z(s-\tau)) d s \\
& \leq V\left(X_{0}\right)+2 L_{k} T \sqrt{K_{1}(k) \Delta t}+\tau L_{k} L \sqrt{\Delta t} \\
& \quad+\mathbf{E} \int_{0}^{t \wedge \rho_{k}}\left(\mu_{1}(1+V(\bar{X}(s))+V(\bar{X}(s-\tau))+U(\bar{X}(s-\tau)))-\mu_{2} U(\bar{X}(s))\right) d s
\end{aligned}
$$

for $0 \leq t \leq T$ and $k \geq[L \sqrt{\tau}+|\xi(0)|]+1$.

Step 2. For $0 \leq t \leq \tau$ and $k \geq[L \sqrt{\tau}+|\xi(0)|]+1$, it follows from (3.18) that

$$
\begin{aligned}
\mathrm{E} V\left(\bar{X}\left(t \wedge \rho_{k}\right)\right) \leq & V\left(X_{0}\right)+2 L_{k} T \sqrt{K_{1}(k) \Delta t}+\tau L_{k} L \sqrt{\Delta t}+\alpha_{1} \\
& +\mu_{1} \mathbf{E} \int_{0}^{t \wedge \rho_{k}} V(\bar{X}(s)) d s-\mu_{2} \mathbf{E} \int_{0}^{t \wedge \rho_{k}} U(\bar{X}(s)) d s
\end{aligned}
$$

where $\alpha_{1}=\mu_{1} \int_{-\tau}^{0}(1+V(\xi(s))+U(\xi(s))) d s$. Thus from (3.19), we get

$$
\begin{aligned}
\mathrm{E} V\left(\bar{X}\left(t \wedge \rho_{k}\right)\right) \leq & V\left(X_{0}\right)+2 L_{k} T \sqrt{K_{1}(k) \Delta t} \\
& +\tau L_{k} L \sqrt{\Delta t}+\alpha_{1}+\mu_{1} \mathrm{E} \int_{0}^{t} V\left(\bar{X}\left(s \wedge \rho_{k}\right)\right) d s,
\end{aligned}
$$

by the Gronwall inequality (see [18]), which gives

$$
\mathbf{E} V\left(\bar{X}\left(t \wedge \rho_{k}\right)\right) \leq\left(V\left(X_{0}\right)+2 L_{k} T \sqrt{K_{1}(k) \Delta t}+\tau L_{k} L \sqrt{\Delta t}+\alpha_{1}\right) e^{\mu_{1} t},
$$

for $0 \leq t \leq \tau$ and $k \geq[L \sqrt{\tau}+|\xi(0)|]+1$. Moreover, from (3.19) and (3.21), we have

$$
\begin{aligned}
& \mathrm{E} \int_{0}^{\tau \wedge \rho_{k}} U(\bar{X}(s)) d s \\
& \quad \leq \mu_{2}^{-1}\left(V\left(X_{0}\right)+2 L_{k} T \sqrt{K_{1}(k) \Delta t}+\tau L_{k} L \sqrt{\Delta t}+\alpha_{1}+\mu_{1} \int_{0}^{\tau} \mathrm{E} V\left(\bar{X}\left(s \wedge \rho_{k}\right)\right) d s\right) \\
& \quad \leq\left(V\left(X_{0}\right)+2 L_{k} T \sqrt{K_{1}(k) \Delta t}+\tau L_{k} L \sqrt{\Delta t}+\alpha_{1}\right) e^{\mu_{1} \tau} \mu_{2}^{-1},
\end{aligned}
$$

for $k \geq[L \sqrt{\tau}+|\xi(0)|]+1$ 
Step 3. For $0 \leq t \leq 2 \tau$ and $k \geq[L \sqrt{\tau}+|\xi(0)|]+1$, it follows from (3.18) that

$$
\begin{aligned}
& \mathrm{EV}\left(\bar{X}\left(t \wedge \rho_{k}\right)\right) \\
& \leq V\left(X_{0}\right)+2 L_{k} T \sqrt{K_{1}(k) \Delta t}+\tau L_{k} L \sqrt{\Delta t}+\alpha_{2} \\
& \quad+\mu_{1} \mathbf{E} \int_{0}^{t \wedge \rho_{k}} V(\bar{X}(s)) d s-\mu_{2} \mathbf{E} \int_{0}^{t \wedge \rho_{k}} U(\bar{X}(s)) d s .
\end{aligned}
$$

As the same way as Step 2, we can obtain

$$
\begin{aligned}
& \mathrm{EV}\left(\bar{X}\left(t \wedge \rho_{k}\right)\right) \\
& \quad \leq\left(V\left(X_{0}\right)+2 L_{k} T \sqrt{K_{1}(k) \Delta t}+\tau L_{k} L \sqrt{\Delta t}+\alpha_{2}\right) e^{\mu_{1} t}, \quad 0 \leq t \leq 2 \tau, \\
& \quad \mathbf{E} \int_{0}^{2 \tau \wedge \rho_{k}} U(\bar{X}(s)) d s \\
& \quad \leq\left(V\left(X_{0}\right)+2 L_{k} T \sqrt{K_{1}(k) \Delta t}+\tau L_{k} L \sqrt{\Delta t}+\alpha_{2}\right) e^{2 \mu_{1} \tau} \mu_{2}^{-1},
\end{aligned}
$$

where

$$
\begin{aligned}
\alpha_{2} & =\mu_{1} \mathbf{E} \int_{0}^{2 \tau \wedge \rho_{k}}(1+V(\bar{X}(s-\tau))+U(\bar{X}(s-\tau))) d s \\
& =\mu_{1} \mathbf{E} \int_{-\tau}^{2 \tau \wedge \rho_{k}-\tau}(1+V(\bar{X}(s))+U(\bar{X}(s))) d s \\
& \leq \mu_{1} \mathbf{E} \int_{-\tau}^{\tau \wedge \rho_{k}}(1+V(\bar{X}(s))+U(\bar{X}(s))) d s \\
& =\alpha_{1}+\mu_{1} \mathbf{E} \int_{0}^{\tau \wedge \rho_{k}}(1+V(\bar{X}(s))+U(\bar{X}(s))) d s \\
& \leq \alpha_{1}+\mu_{1} \tau+\mu_{1} \int_{0}^{\tau} \mathbf{E} V\left(\bar{X}\left(s \wedge \rho_{k}\right)\right) d s+\mu_{1} \mathbf{E} \int_{0}^{\tau \wedge \rho_{k}} U(\bar{X}(s)) d s \\
& \leq \mu_{1} \tau+\left(V\left(X_{0}\right)+2 L_{k} T \sqrt{K_{1}(k) \Delta t}+\tau L_{k} L \sqrt{\Delta t}+\alpha_{1}\right)\left(e^{\mu_{1} \tau}+e^{\mu_{1} \tau} \mu_{1} \mu_{2}^{-1}\right),
\end{aligned}
$$

from (3.21) and (3.22). So (3.24) becomes

$$
\mathbf{E} V\left(\bar{X}\left(t \wedge \rho_{k}\right)\right) \leq\left(V\left(X_{0}\right)+2 L_{k} T \sqrt{K_{1}(k) \Delta t}+\tau L_{k} L \sqrt{\Delta t}+\alpha_{1}\right) \beta_{1,0}+\beta_{2,0},
$$

for $0 \leq t \leq 2 \tau$ and $k \geq[L \sqrt{\tau}+|\xi(0)|]+1$, where

$$
\beta_{1,0}=\left(1+e^{\mu_{1} \tau}+e^{\mu_{1} \tau} \mu_{1} \mu_{2}^{-1}\right) e^{2 \mu_{1} \tau}, \quad \beta_{2,0}=\left(\mu_{1} \tau-\alpha_{1}\right) e^{2 \mu_{1} \tau}
$$


Step 4. By repeating the same way in Steps 2 and 3, we get

$$
\mathbf{E V}\left(\bar{X}\left(T \wedge \rho_{k}\right)\right) \leq\left(V\left(X_{0}\right)+2 L_{k} T \sqrt{K_{1}(k) \Delta t}+\tau L_{k} L \sqrt{\Delta t}+\alpha_{1}\right) \beta_{1}+\beta_{2}
$$

for $k \geq[L \sqrt{\tau}+|\xi(0)|]+1$, where $\beta_{1}$ and $\beta_{2}$ are two constants dependent on $\mu_{1}, \mu_{2}, \tau, T$ and independent of $k$ and $\Delta t$. Therefore, we have

$$
\mathbf{P}\left(\rho_{k} \leq T\right) \leq \frac{V\left(X_{0}\right) \beta_{1}+\alpha_{1} \beta_{1}+\beta_{2}+2 \beta_{1} L_{k} T \sqrt{K_{1}(k) \Delta t}+\beta_{1} \tau L_{k} L \sqrt{\Delta t}}{\vartheta_{k}}
$$

where

$$
\vartheta_{k}=\inf _{|\bar{X}| \geq k} V(\bar{X}), \quad k \geq[L \sqrt{\tau}+|\xi(0)|]+1
$$

Now, for any $\epsilon \in(0,1)$, we can choose sufficiently large integer $k^{*}$ such that

$$
\frac{V\left(X_{0}\right) \beta_{1}+\alpha_{1} \beta_{1}+\beta_{2}}{\vartheta_{k^{*}}} \leq \frac{\epsilon}{2}
$$

and sufficiently small $\Delta t_{1}^{*}$ such that

$$
\frac{2 \beta_{1} L_{k^{*}} T \sqrt{K_{1}\left(k^{*}\right) \Delta t_{1}^{*}}+\beta_{1} \tau L_{k^{*}} L \sqrt{\Delta t_{1}^{*}}}{\vartheta_{k^{*}}} \leq \frac{\epsilon}{2} .
$$

So from (3.30), we can obtain

$$
\mathbf{P}\left(\rho_{k^{*}} \leq T\right) \leq \epsilon, \quad \forall \Delta t \leq \Delta t_{1}^{*}
$$

\section{Convergence in Probability}

In this section, we show the convergence in probability of the Euler method to (2.1) over a finite time interval $[0, T]$, which is based on the following lemma.

Lemma 4.1. Under Assumptions 2.1, 2.3, and 2.4, for any $T>0$, there exists a positive constant $K_{2}(k)$, dependent on $k$ and independent of $\Delta t$, such that for all $\Delta t \in(0,1)$ the solution of $(2.1)$ and the continuous-time Euler method (3.4) satisfy

$$
\mathbf{E}\left(\sup _{0 \leq t \leq T}\left|x\left(t \wedge \sigma_{k} \wedge \rho_{k}\right)-\bar{X}\left(t \wedge \sigma_{k} \wedge \rho_{k}\right)\right|^{2}\right) \leq K_{2}(k) \Delta t
$$

where $\sigma_{k}$ and $\rho_{k}$ are defined in Lemmas 2.7 and 3.1, respectively, and $k \geq[L \sqrt{\tau}+|\xi(0)|]+1$. 
Proof. From (2.1) and (3.4), for any $0 \leq t^{\prime} \leq T$ and $k \geq[L \sqrt{\tau}+|\xi(0)|]+1$, we have

$$
\begin{aligned}
& \mathrm{E}\left(\sup _{0 \leq t \leq t^{\prime}}\left|x\left(t \wedge \sigma_{k} \wedge \rho_{k}\right)-\bar{X}\left(t \wedge \sigma_{k} \wedge \rho_{k}\right)\right|^{2}\right) \\
& \leq 3 \mathrm{E}\left(\sup _{0 \leq t \leq t^{\prime}}\left|\int_{0}^{t \wedge \sigma_{k} \wedge \rho_{k}}\left(a\left(x\left(s^{-}\right), x\left((s-\tau)^{-}\right)\right)-a(Z(s), Z(s-\tau))\right) d s\right|^{2}\right) \\
& \quad+3 \mathrm{E}\left(\sup _{0 \leq t \leq t^{\prime}}\left|\int_{0}^{t \wedge \sigma_{k} \wedge \rho_{k}}\left(b\left(x\left(s^{-}\right), x\left((s-\tau)^{-}\right)\right)-b(Z(s), Z(s-\tau))\right) d W(s)\right|^{2}\right) \\
& \quad+3 \mathrm{E}\left(\sup _{0 \leq t \leq t^{\prime}}\left|\int_{0}^{t \wedge \sigma_{k} \wedge \rho_{k}} \int_{\varepsilon}\left(c\left(x\left(s^{-}\right), x\left((s-\tau)^{-}\right), v\right)-c(Z(s), Z(s-\tau), v)\right) \tilde{p}_{\phi}(d v \times d s)\right|^{2}\right),
\end{aligned}
$$

where the inequality $\left|u_{1}+u_{2}+u_{3}\right|^{2} \leq 3\left|u_{1}\right|^{2}+3\left|u_{2}\right|^{2}+3\left|u_{3}\right|^{2}$ for $u_{1}, u_{2}, u_{3} \in \mathbf{R}^{d}$ is used. Therefore, by using the Cauchy-Schwarz inequality, Assumptions 2.1 and 2.4, Fubini's Theorem, and Lemma 3.1, we obtain

$$
\begin{aligned}
& \mathbf{E}\left(\sup _{0 \leq t \leq t^{\prime}}\left|\int_{0}^{t \wedge \sigma_{k} \wedge \rho_{k}}\left(a\left(x\left(s^{-}\right), x\left((s-\tau)^{-}\right)\right)-a(Z(s), Z(s-\tau))\right) d s\right|^{2}\right) \\
& \leq \mathbf{E}\left(\sup _{0 \leq t \leq t^{\prime}} \int_{0}^{t \wedge \sigma_{k} \wedge \rho_{k}} 1^{2} d s \int_{0}^{t \wedge \sigma_{k} \wedge \rho_{k}}\left|a\left(x\left(s^{-}\right), x\left((s-\tau)^{-}\right)\right)-a(Z(s), Z(s-\tau))\right|^{2} d s\right) \\
& \leq T \mathbf{E}\left(\int_{0}^{t^{\prime} \wedge \sigma_{k} \wedge \rho_{k}}\left|a\left(x\left(s^{-}\right), x\left((s-\tau)^{-}\right)\right)-a(Z(s), Z(s-\tau))\right|^{2} d s\right) \\
& \leq T C_{k} \mathbf{E}\left(\int_{0}^{t^{\prime} \wedge \sigma_{k} \wedge \rho_{k}}\left|x\left(s^{-}\right)-Z(s)\right|^{2} d s\right)+T C_{k} \mathbf{E}\left(\int_{0}^{t^{\prime} \wedge \sigma_{k} \wedge \rho_{k}}\left|x\left((s-\tau)^{-}\right)-Z(s-\tau)\right|^{2} d s\right) \\
& \leq 2 T C_{k} \mathbf{E}\left(\int_{0}^{t^{\prime} \wedge \sigma_{k} \wedge \rho_{k}}\left|x\left(s^{-}\right)-Z(s)\right|^{2} d s\right)+T C_{k} \int_{-\tau}^{0}|\xi(s)-Z(s)|^{2} d s \\
& \leq 4 T C_{k} \mathbf{E}\left(\int_{0}^{t^{\prime} \wedge \sigma_{k} \wedge \rho_{k}}|\bar{X}(s)-Z(s)|^{2} d s\right) \\
& \quad+4 T C_{k} \mathbf{E}\left(\int_{0}^{t^{\prime} \wedge \sigma_{k} \wedge \rho_{k}}\left|x\left(s^{-}\right)-\bar{X}(s)\right|^{2} d s\right) \\
& \quad+T C_{k} \sum_{n=-m}^{-1} \int_{t_{n}}^{t_{n+1}}\left|\xi(s)-\xi\left(t_{n}\right)\right|^{2} d s
\end{aligned}
$$


Abstract and Applied Analysis

$$
\begin{aligned}
\leq & 4 T C_{k}\left(\int_{0}^{t^{\prime}} \mathrm{E}\left|\bar{X}\left(s \wedge \sigma_{k} \wedge \rho_{k}\right)-Z\left(s \wedge \sigma_{k} \wedge \rho_{k}\right)\right|^{2} d s\right) \\
& +4 T C_{k} \int_{0}^{t^{\prime}} \mathrm{E}\left(\sup _{0 \leq u \leq s}\left|x\left(u \wedge \sigma_{k} \wedge \rho_{k}^{-}\right)-\bar{X}\left(u \wedge \sigma_{k} \wedge \rho_{k}\right)\right|^{2}\right) d s+T C_{k} L^{2} \tau \Delta t \\
\leq & \left(4 T^{2} C_{k} K_{1}(k)+T C_{k} L^{2} \tau\right) \Delta t \\
& +4 T C_{k} \int_{0}^{t^{\prime}} \mathrm{E}\left(\sup _{0 \leq u \leq s}\left|x\left(u \wedge \sigma_{k} \wedge \rho_{k}^{-}\right)-\bar{X}\left(u \wedge \sigma_{k} \wedge \rho_{k}\right)\right|^{2}\right) d s .
\end{aligned}
$$

Moreover, by using the martingale properties of $d W(t)$ and $\tilde{p}_{\phi}(d v \times d t)$, Assumptions 2.1 and 2.4, Fubini's Theorem, and Lemma 3.1, we have

$$
\begin{aligned}
& \mathbf{E}\left(\sup _{0 \leq t \leq t^{\prime}}\left|\int_{0}^{t \wedge \sigma_{k} \wedge \rho_{k}}\left(b\left(x\left(s^{-}\right), x\left((s-\tau)^{-}\right)\right)-b(Z(s), Z(s-\tau))\right) d W(s)\right|^{2}\right) \\
& \leq 4 \mathbf{E} \int_{0}^{t^{\prime} \wedge \sigma_{k} \wedge \rho_{k}}\left|b\left(x\left(s^{-}\right), x\left((s-\tau)^{-}\right)\right)-b(Z(s), Z(s-\tau))\right|^{2} d s \\
& \leq 4 C_{k} \mathbf{E} \int_{0}^{t^{\prime} \wedge \sigma_{k} \wedge \rho_{k}}\left|x\left(s^{-}\right)-Z(s)\right|^{2} d s+4 C_{k} \mathbf{E} \int_{0}^{t^{\prime} \wedge \sigma_{k} \wedge \rho_{k}}\left|x\left((s-\tau)^{-}\right)-Z(s-\tau)\right|^{2} d s \\
& \leq 8 C_{k} \mathbf{E} \int_{0}^{t^{\prime} \wedge \sigma_{k} \wedge \rho_{k}}\left|x\left(s^{-}\right)-Z(s)\right|^{2} d s+4 C_{k} \int_{-\tau}^{0}|\xi(s)-Z(s)|^{2} d s \\
& \leq 16 C_{k} \mathbf{E}\left(\int_{0}^{t^{\prime} \wedge \sigma_{k} \wedge \rho_{k}}|\bar{X}(s)-Z(s)|^{2} d s\right)+16 C_{k} \mathbf{E}\left(\int_{0}^{t^{\prime} \wedge \sigma_{k} \wedge \rho_{k}}\left|x\left(s^{-}\right)-\bar{X}(s)\right|^{2} d s\right) \\
& \quad+4 C_{k} \sum_{n=-m}^{-1} \int_{t_{n}}^{t_{n+1}}\left|\xi(s)-\xi\left(t_{n}\right)\right|^{2} d s \\
& \quad \leq\left(16 T C_{k} K_{1}(k)+4 C_{k} L^{2} \tau\right) \Delta t \\
& \quad+16 C_{k} \int_{0}^{t^{\prime}} \mathbf{E}\left(\sup _{0 \leq u \leq s}\left|x\left(u \wedge \sigma_{k} \wedge \rho_{k}^{-}\right)-\bar{X}\left(u \wedge \sigma_{k} \wedge \rho_{k}\right)\right|^{2}\right) d s, \\
& \left.\quad \sup _{0 \leq t \leq t^{\prime}}\left|\int_{0}^{t \wedge \sigma_{k} \wedge \rho_{k}} \int_{\varepsilon}\left(c\left(x\left(s^{-}\right), x\left((s-\tau)^{-}\right), v\right)-c(Z(s), Z(s-\tau), v)\right) \tilde{p}_{\phi}(d v \times d s)\right|^{2}\right)
\end{aligned}
$$




$$
\begin{aligned}
\leq & 4 \mathrm{E}\left|\int_{0}^{t^{\prime} \wedge \sigma_{k} \wedge \rho_{k}} \int_{\varepsilon}\left(c\left(x\left(s^{-}\right), x\left((s-\tau)^{-}\right), v\right)-c(Z(s), Z(s-\tau), v)\right) \tilde{p}_{\phi}(d v \times d s)\right|^{2} \\
= & 4 \mathrm{E} \int_{0}^{t^{\prime} \wedge \sigma_{k} \wedge \rho_{k}} \int_{\varepsilon}\left|c\left(x\left(s^{-}\right), x\left((s-\tau)^{-}\right), v\right)-c(Z(s), Z(s-\tau), v)\right|^{2} \phi(d v) d s \\
\leq & \left(16 T C K_{1}(k)+4 C L^{2} \tau\right) \Delta t \\
& +16 C \int_{0}^{t^{\prime}} \mathrm{E}\left(\sup _{0 \leq u \leq s}\left|x\left(u \wedge \sigma_{k} \wedge \rho_{k}^{-}\right)-\bar{X}\left(u \wedge \sigma_{k} \wedge \rho_{k}\right)\right|^{2}\right) d s .
\end{aligned}
$$

Therefore by substituting (4.3) and (4.4) into (4.2), we get

$$
\begin{aligned}
& \mathbf{E}\left(\sup _{0 \leq t \leq t^{\prime}}\left|x\left(t \wedge \sigma_{k} \wedge \rho_{k}\right)-\bar{X}\left(t \wedge \sigma_{k} \wedge \rho_{k}\right)\right|^{2}\right) \\
& \quad \leq \Delta t\left(12 T^{2} C_{k} K_{1}(k)+3 T C_{k} L^{2} \tau+48 T C_{k} K_{1}(k)+48 T C K_{1}(k)+12 C_{k} L^{2} \tau+12 C L^{2} \tau\right) \\
& \quad+\left(12 T C_{k}+48 C_{k}+48 C\right) \int_{0}^{t^{\prime}} \mathbf{E}\left(\sup _{0 \leq u \leq s}\left|x\left(u \wedge \sigma_{k} \wedge \rho_{k}^{-}\right)-\bar{X}\left(u \wedge \sigma_{k} \wedge \rho_{k}\right)\right|^{2}\right) d s .
\end{aligned}
$$

So by using the Gronwall inequality (see [18]), we have the result (4.1) by choosing

$$
\begin{aligned}
K_{2}(k)=( & 12 T^{2} C_{k} K_{1}(k)+3 T C_{k} L^{2} \tau+48 T C_{k} K_{1}(k)+48 T C K_{1}(k) \\
& \left.+12 C_{k} L^{2} \tau+12 C L^{2} \tau\right) \exp \left(12 T^{2} C_{k}+48 T C_{k}+48 T C\right) .
\end{aligned}
$$

Now, we state our main theorem which shows the convergence in probability of the continuous-time Euler method (3.4).

Theorem 4.2. Under Assumptions 2.1, 2.2, 2.3, and 2.4, for sufficiently small $\epsilon, \varsigma \in(0,1)$, there is a $\Delta t^{*}$ such that for all $\Delta t<\Delta t^{*}$

$$
\mathbf{P}\left(\sup _{0 \leq t \leq T}|x(t)-\bar{X}(t)|^{2} \geq \varsigma\right) \leq \epsilon
$$

for any $T>0$.

Proof. For sufficiently small $\epsilon, \varsigma \in(0,1)$, we define

$$
\bar{\Omega}=\left\{\omega: \sup _{0 \leq t \leq T}|x(t)-\bar{X}(t)|^{2} \geq \varsigma\right\}
$$


By Lemmas 2.9 and 3.2, there exists a pair of $k^{\star}$ and $\Delta t_{1}^{\star}$ such that

$$
\begin{gathered}
\mathbf{P}\left(\sigma_{k^{\star}} \leq T\right) \leq \frac{\epsilon}{3}, \\
\mathbf{P}\left(\rho_{k^{\star}} \leq T\right) \leq \frac{\epsilon}{3}, \quad \forall \Delta t \leq \Delta t_{1}^{\star} .
\end{gathered}
$$

We then have

$$
\begin{aligned}
\mathbf{P}(\bar{\Omega}) & \leq \mathbf{P}\left(\bar{\Omega} \cap\left\{\sigma_{k^{\star}} \wedge \rho_{k^{\star}}>T\right\}\right)+\mathbf{P}\left(\sigma_{k^{\star}} \wedge \sigma_{k^{\star}} \leq T\right) \\
& \leq \mathbf{P}\left(\bar{\Omega} \cap\left\{\sigma_{k^{\star}} \wedge \sigma_{k^{\star}}>T\right\}\right)+\mathbf{P}\left(\sigma_{k^{\star}} \leq T\right)+\mathbf{P}\left(\rho_{k^{\star}} \leq T\right) \\
& \leq \mathbf{P}\left(\bar{\Omega} \cap\left\{\sigma_{k^{\star}} \wedge \rho_{k^{\star}}>T\right\}\right)+\frac{2 \epsilon}{3},
\end{aligned}
$$

for $\Delta t \leq \Delta t_{1}^{\star}$. Moreover, from Lemma 4.1, we have

$$
\begin{aligned}
{ }_{S} \mathbf{P}\left(\bar{\Omega} \cap\left\{\sigma_{k^{\star}} \wedge \rho_{k^{\star}}>T\right\}\right) & \leq \mathbf{E}\left(I_{\left\{\sigma_{k^{\star}} \wedge \rho_{k^{\star}}>T\right\}} \sup _{0 \leq t \leq T}|x(t)-\bar{X}(t)|^{2}\right) \\
& \leq \mathbf{E}\left(\sup _{0 \leq t \leq T}\left|x\left(t \wedge \sigma_{k^{\star}} \wedge \rho_{k^{\star}}\right)-\bar{X}\left(t \wedge \sigma_{k^{\star}} \wedge \rho_{k^{\star}}\right)\right|^{2}\right) \\
& \leq K_{2}\left(k^{\star}\right) \Delta t,
\end{aligned}
$$

which gives

$$
\mathbf{P}\left(\bar{\Omega} \cap\left\{\sigma_{k^{\star}} \wedge \rho_{k^{\star}}>T\right\}\right) \leq \frac{\epsilon}{3}
$$

for $\Delta t \leq \Delta t_{2}^{\star}$. Hence, from the inequalities above, we get

$$
\mathbf{P}(\bar{\Omega}) \leq \epsilon,
$$

for $\Delta t \leq \Delta t^{\star}$, where $\Delta t^{\star}=\min \left\{\Delta t_{1}^{\star}, \Delta t_{2}^{\star}\right\}$.

We remark that the continuous-time Euler solution $\bar{X}(t)$ cannot be computed, since it requires knowledge of the entire Brownian motion and Poisson random measure paths. So the last theorem demonstrates the convergence in probability of the discrete Euler solution (3.2). 
Theorem 4.3. Under Assumptions 2.1, 2.2, 2.3, and 2.4, for sufficiently small $\epsilon, \varsigma \in(0,1)$, there is a $\Delta t^{*}$ such that for all $\Delta t<\Delta t^{*}$

$$
\mathbf{P}\left(|x(t)-Z(t)|^{2} \geq \varsigma, 0 \leq t \leq T\right) \leq \epsilon,
$$

for any $T>0$.

Proof. For sufficiently small $\epsilon, \varsigma \in(0,1)$, we define

$$
\widetilde{\Omega}=\left\{\omega:|x(t)-Z(t)|^{2} \geq \varsigma, 0 \leq t \leq T\right\} .
$$

As the same analysis as the proof in Theorem 4.2, we have

$$
\mathbf{P}(\tilde{\Omega}) \leq \mathbf{P}\left(\tilde{\Omega} \cap\left\{\sigma_{k^{\star}} \wedge \rho_{k^{\star}}>T\right\}\right)+\frac{2 \epsilon}{3} .
$$

Recalling that

$$
\begin{aligned}
{ }_{S} \mathbf{P}\left(\widetilde{\Omega} \cap\left\{\sigma_{k^{\star}} \wedge \rho_{k^{\star}}>T\right\}\right) \leq & \mathbf{E}\left(|x(T)-Z(T)|^{2} I_{\left\{\sigma_{k^{\star}} \wedge \rho_{k^{\star}}>T\right\}}\right) \\
\leq & \mathbf{E}\left|x\left(T \wedge \sigma_{k^{\star}} \wedge \rho_{k^{\star}}\right)-Z\left(T \wedge \sigma_{k^{\star}} \wedge \rho_{k^{\star}}\right)\right|^{2} \\
\leq & 2 \mathrm{E}\left(\sup _{0 \leq t \leq T}\left|x\left(t \wedge \sigma_{k^{\star}} \wedge \rho_{k^{\star}}\right)-\bar{X}\left(t \wedge \sigma_{k^{\star}} \wedge \rho_{k^{\star}}\right)\right|^{2}\right) \\
& +2 \mathrm{E}\left|\bar{X}\left(T \wedge \sigma_{k^{\star}} \wedge \rho_{k^{\star}}\right)-Z\left(T \wedge \sigma_{k^{\star}} \wedge \rho_{k^{\star}}\right)\right|^{2} \\
\leq & 2 K_{1}\left(k^{\star}\right) \Delta t+2 K_{2}\left(k^{\star}\right) \Delta t
\end{aligned}
$$

and using Lemmas 3.1 and 4.1, we obtain

$$
\mathbf{P}\left(\widetilde{\Omega} \cap\left\{\sigma_{k^{\star}} \wedge \rho_{k^{\star}}>T\right\}\right) \leq \frac{\epsilon}{3},
$$

for sufficiently small $\Delta t$. So the inequalities above demonstrate

$$
\mathbf{P}(\tilde{\Omega}) \leq \epsilon
$$

for all sufficiently small $\Delta t$.

Hence we complete the result (4.14).

\section{Numerical Example}

In this section, a numerical example is analyzed under Assumptions 2.1, 2.2, 2.3, and 2.4 which cover many highly nonlinear SDDEs driven by Poisson random measure. 
Now, we consider the following equation:

$$
\begin{aligned}
d x(t)= & \left(2 x^{2}\left((t-0.05)^{-}\right)-4 x^{3}\left(t^{-}\right)\right) d t+3 x^{2}\left((t-0.05)^{-}\right) d W(t) \\
& +\int_{\varepsilon} v x^{2}\left((t-0.05)^{-}\right) \tilde{p}_{\phi}(d v \times d t), \quad t>0
\end{aligned}
$$

with $x(t)=t^{2}, t \in[-0.05,0]$, where $d=m=r=1$. The compensated measure of the Poisson random measure $p_{\phi}(d v \times d t)$ is given by $\phi(d v) d t=\lambda f(v) d v d t$, where $\lambda=5$ and

$$
f(v)=\frac{1}{\sqrt{2 \pi} v} \exp \left(-\frac{(\ln v)^{2}}{2}\right), \quad 0 \leq v<\infty,
$$

is the density function of a lognormal random variable.

Clearly, the equation cannot satisfy the global Lipschitz conditions, the linear growth conditions and the classical Khasminskii-type conditions. But, the local Lipschitz conditions are satisfied. On the other hand, for $V(x)=|x|^{2}$, we have

$$
\begin{aligned}
L V(x, y) & =2 x\left(2 y^{2}-4 x^{3}\right)+9 y^{4}+\int_{\varepsilon} v^{2} y^{4} \lambda \frac{1}{\sqrt{2 \pi} v} \exp \left(-\frac{(\ln v)^{2}}{2}\right) d v \\
& \leq 2 x^{2}+60 y^{4}-8 x^{4} \\
& =60\left(1+x^{2}+y^{2}+y^{4}\right)-8 x^{4},
\end{aligned}
$$

where $U(x)=x^{4}, \mu_{1}=60, \mu_{2}=8$. In other words, the equation satisfies Assumptions 2.1, 2.2, 2.3, and 2.4.

So according to Theorem 2.8, (5.1) has a unique global solution $x(t)$ on $t \in[-0.05, \infty)$.

Given the stepsize $\Delta t=0.0025$, we can have the Euler method to (5.1)

$$
\begin{gathered}
X_{n}=(n \Delta t)^{2}, \quad \text { for } n=-20,-19, \ldots,-1,0, \\
X_{n+1}=X_{n}+\left(2 X_{n-20}^{2}-4 X_{n}^{3}\right) \Delta t+3 X_{n-20}^{2} \Delta W_{n}+\int_{t_{n}}^{t_{n+1}} \int_{\varepsilon} v X_{n-20}^{2} \tilde{p}_{\phi}(d v \times d t),
\end{gathered}
$$

for $n=0,1, \ldots$, where $\Delta W_{n}=W\left(t_{n+1}\right)-W\left(t_{n}\right)$.

And in Matlab experiment, we actually obtain the discrete Euler

$$
\begin{aligned}
X_{n+1}= & X_{n}+\left(2 X_{n-20}^{2}-4 X_{n}^{3}-5 \sqrt{e} X_{n-20}^{2}\right) \Delta t \\
& +3 X_{n-20}^{2} \Delta W_{n}+X_{n-20}^{2} \sum_{i=p_{\phi}\left(t_{n}\right)+1}^{p_{\phi}\left(t_{n+1}\right)} \xi_{i}
\end{aligned}
$$

where $\xi_{i}$ is distributed according to $f(v)$. Subsequently, we can get the result in Theorems 4.2 and 4.3. 


\section{Acknowledgment}

This work is supported by the NSF of China (no. 11071050).

\section{References}

[1] P. J. Schönbucher, Credit Derivatives Pricing Models: Models, Pricing and Implementation, John Wiley \& Sons, Chichester, UK, 2003.

[2] R. Cont and P. Tankov, Financial Modelling with Jump Processes, Financial Mathematics Series, Chapman \& Hall, London, UK, 2004.

[3] R. C. Merton, "Option pricing when underlying stock returns are discontinuous," Journal of Financial Economics, vol. 3, no. 1-2, pp. 125-144, 1976.

[4] W. Mao, "Convergence of numerical solutions for variable delay differential equations driven by Poisson random jump measure," Applied Mathematics and Computation, vol. 212, no. 2, pp. 409-417, 2009.

[5] R. H. Liu, H. B. Meng, and Y. H. Dai, "Convergence of numerical solutions to stochastic delay differential equations with jumps," Applied Mathematics and Computation, vol. 172, no. 1, pp. 584-602, 2006.

[6] X. Q. Liu and C. W. Li, "Weak approximation and extrapolations of stochastic differential equations with jumps," SIAM Journal on Numerical Analysis, vol. 37, no. 6, pp. 1747-1767, 2000.

[7] N. Bruti-Liberati and E. Platen, "Approximation of jump diffusions in finance and economics," Computational Economics, vol. 29, no. 3-4, pp. 283-312, 2007.

[8] E. Mordecki, A. Szepessy, R. Tempone, and G. E. Zouraris, "Adaptive weak approximation of diffusions with jumps," SIAM Journal on Numerical Analysis, vol. 46, no. 4, pp. 1732-1768, 2008.

[9] E. Platen, "An approximation method for a class of Itô processes with jump component," Lietuvos Matematikos Rinkinys, vol. 22, no. 2, pp. 124-136, 1982a.

[10] N. Bruti-Liberati and E. Platen, “On the strong approximation of jump-diffusion processes," Tech. Rep. Quantitative Finance Research Papers 157, University of Technology, Sydney, Australia, 2005.

[11] N. Bruti-Liberati and E. Platen, "Strong approximations of stochastic differential equations with jumps," Journal of Computational and Applied Mathematics, vol. 205, no. 2, pp. 982-1001, 2007.

[12] E. Buckwar and M. G. Riedler, "Runge-Kutta methods for jump-diffusion differential equations," Journal of Computational and Applied Mathematics, vol. 236, no. 6, pp. 1155-1182, 2011.

[13] R. Z. Khasminskii, Stochastic Stability of Differential Equations, Sijthoff and Noordhoff, Alphen aan den Rijn, The Netherlands, 1981.

[14] X. R. Mao and M. J. Rassias, "Khasminskii-type theorems for stochastic differential delay equations," Stochastic Analysis and Applications, vol. 23, no. 5, pp. 1045-1069, 2005.

[15] F. Wu, "Khasminskii-type theorems for neutral stochastic functional differential equations," Mathematica Applicata, vol. 21, no. 4, pp. 794-799, 2008.

[16] X. R. Mao, "Numerical solutions of stochastic differential delay equations under the generalized Khasminskii-type conditions," Applied Mathematics and Computation, vol. 217, no. 12, pp. 5512-5524, 2011.

[17] S. Albeverio, Z. Brzeźniak, and J. L. Wu, "Existence of global solutions and invariant measures for stochastic differential equations driven by Poisson type noise with non-Lipschitz coefficients," Journal of Mathematical Analysis and Applications, vol. 371, no. 1, pp. 309-322, 2010.

[18] X. R. Mao and C. G. Yuan, Stochastic Differential Equations with Markovian Switching, Imperial College Press, London, UK, 2006. 


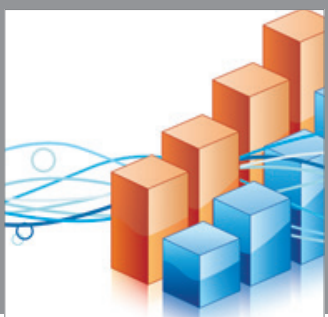

Advances in

Operations Research

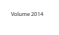

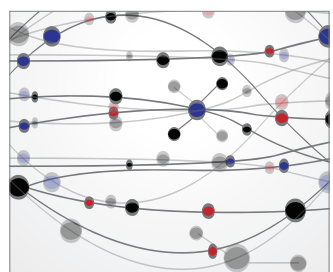

\section{The Scientific} World Journal
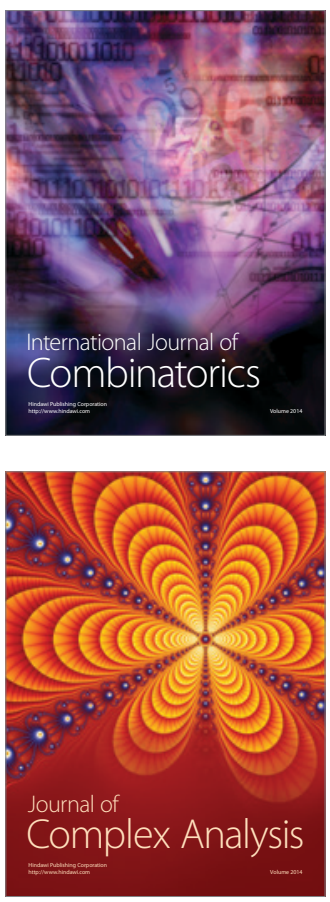

International Journal of

Mathematics and

Mathematical

Sciences
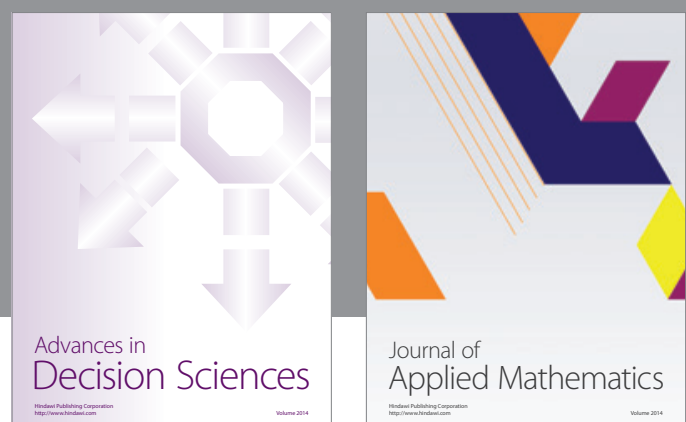

Journal of

Applied Mathematics
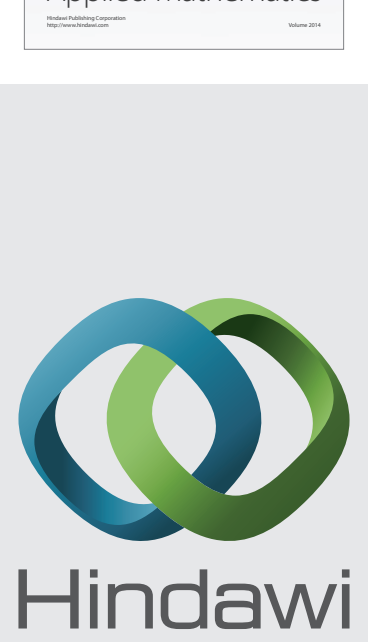

Submit your manuscripts at http://www.hindawi.com
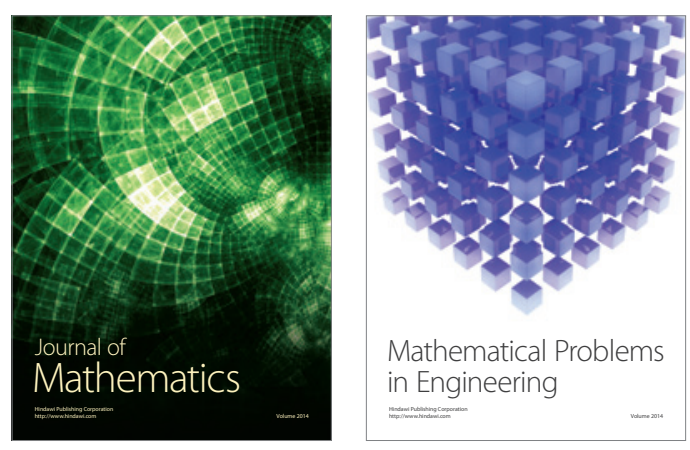

Mathematical Problems in Engineering
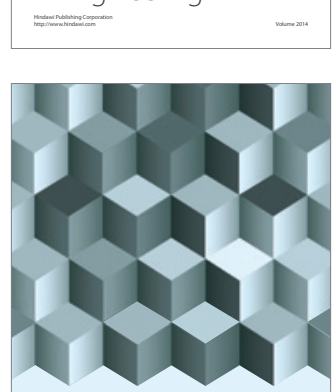

Journal of

Function Spaces
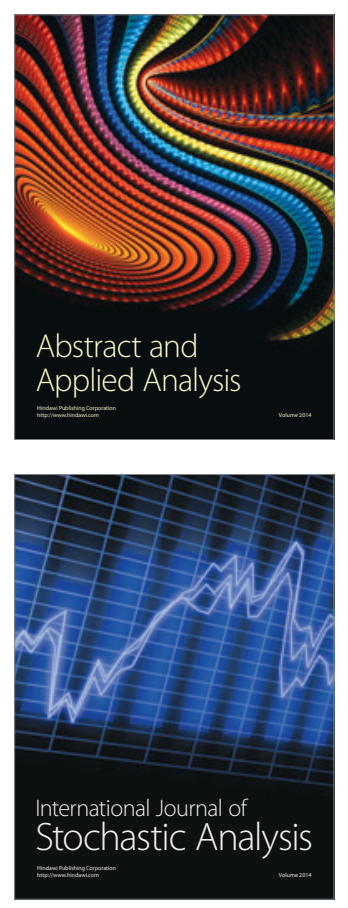

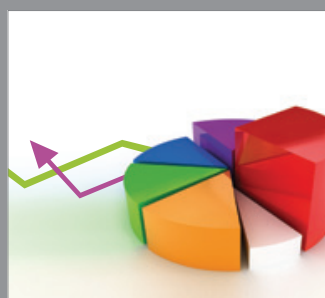

ournal of

Probability and Statistics

Promensencen
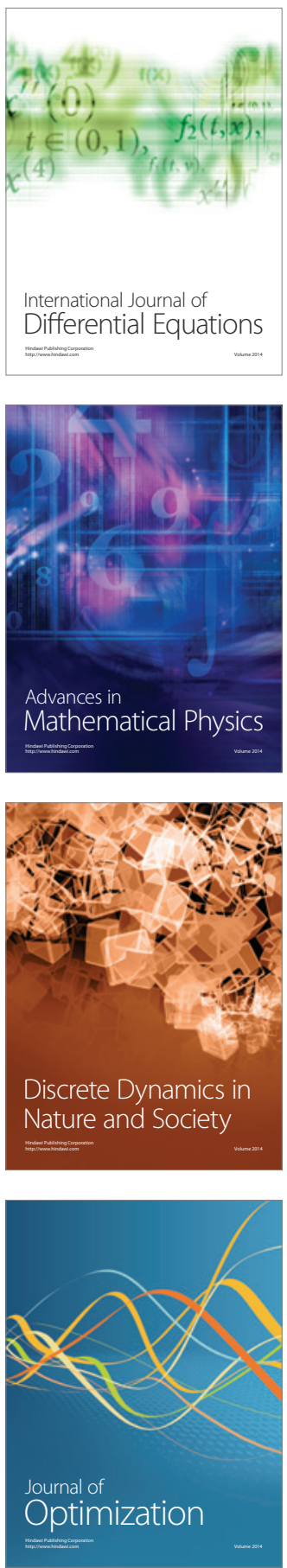